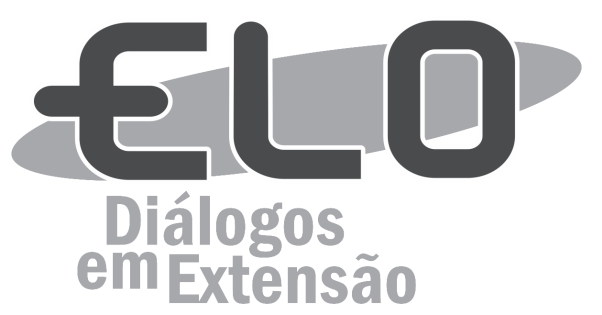

\title{
Avaliação do Ciclo de Aprendizagem Vivencial em uma Cooperativa Agropecuária
}

William Moreira Alves ${ }^{1}$, Guilherme Luís Rosa da Silva ${ }^{1}$, Roberto Max Protil ${ }^{1}$, Pablo Murta Baião Albino ${ }^{1,2}$

\begin{abstract}
Resumo: Este trabalho teve o intuito de avaliar a aplicação de jogos de empresa e a utilização da metodologia do CAV - Ciclo de Aprendizagem vivencial, junto aos cooperados e funcionários de uma cooperativa agropecuária do estado de Minas Gerais. Na interpretação dos resultados foi utilizado o método DSC - Discurso do Sujeito Coletivo, que permitiu sintetizar as informações dos participantes referentes à prática dos jogos de empresas e do CAV ao transmitir as concepções das atividades realizadas por meio de discursos. O CAV possibilitou aos integrantes compartilhar o conhecimento adquirido com a aplicação do jogo de empresas $e$ demonstrar seus diferentes pontos de vistas e sentimentos sobre as atividades realizadas. Inseridos em um ambiente de interação e espontaneidade os participantes puderam expor suas opiniões, desenvolver conhecimentos compartilhados e, por fim, enriquecer o curso por meio de um aprendizado coletivo.
\end{abstract}

Palavras-chave: Ciclo de aprendizagem vivencial. Jogos de empresas. Discurso do sujeito coletivo.

Área Temática: Extensão rural.

\section{Avaliation of the Experiential Learning Cycle in an Agricultural Cooperative}

Abstract: This work aimed to evaluate the application of business games and the use of the methodology $C A V$ - Experiential Learning Cycle, from the members and employees of an agricultural cooperative of the Minas Gerais state. For the results analysis we used the DSC-Collective Subject Discourse method, which allowed synthesize information from participants regarding the practice of business games and $C A V$ to convey the concepts of activities through speeches. The CAV allowed the participants to share the knowledge gained from the application of business games and demonstrate their different views and feelings about the activities carried out by creating an interaction and the spontaneity environment make the participants feel free to express their opinions, developing a shared knowledge, enriching thus the course through a collective learning.

Keywords: Experiential learning cycle. Business games. Discourse of the collective subject.

\section{Evaluátion del Ciclo de Aprendizaje Experiencial en una Cooperativa Agricola}

Resumen: Este trabajo tuvo como objetivo evaluar la aplicación de juegos de negocios y el uso de la metodología CAV-Ciclo de Aprendizaje Experimental con los miembros y empleados de una cooperativa agrícola en el estado de Minas Gerais. En la interpretación de los resultados se utilizó el método DSC-Discurso Colectiva del Sujeto, lo que permitió sintetizar la información de los participantes en cuanto a la práctica de las empresas de juegos y CAV para transmitir los conceptos de actividades a través de discursos. El CAV permitió a los participantes a compartir los conocimientos obtenidos de la aplicación de juegos de empresas y demostrar sus diferentes puntos de vista y sentimientos acerca de las actividades llevadas a cabo mediante la creación de un entorno de interacción

${ }^{1}$ Universidade Federal de Viçosa, Departamento de Economia Rural.

${ }^{2}$ Endereço de contato: DER/UFV Av. Purdue s/n 36570-000 Viçosa/MG, (31)38991319, pablo.albino@ufv.br 
y la espontaneidad lo que hacen los participantes se sientan libres para expresar su opiniones, el desarrollo de un conocimiento compartido, enriqueciendo así el curso a través de un aprendizaje colectivo.

Palabras clave: Ciclo de aprendizaje experimental. Juegos de negocios. Discurso del sujeto colectivo.

\section{Introdução}

O processo de aprendizagem organizacional confronta com diversos fatores administrativos no planejamento e gerenciamento que, por sua vez, podem afetar o desenvolvimento dos objetivos das instituições. Diante disto, ao longo dos anos, diferentes métodos de ensino aprendizagem foram desenvolvidos, possibilitando as organizações prepararem seus colaboradores para estarem aptos às mais diversas situações do mercado.

Uma dessas metodologias aplicadas nas organizações é conhecida como jogos de empresas. Nesses jogos os participantes possuem a oportunidade de aprendizagem vivencial a partir da aplicação de jogos, os quais trabalham com questões que envolvem a administração, planejamento, gestão de tempo, negociação, conflitos e principalmente tomadas de decisões estratégicas. Para Lacruz (2004), os jogos de empresa podem ser entendidos como procedimentos educacionais dinâmicos nos quais os participantes podem alcançar um importante nível de aprendizado por meio de atividades lúdicas, fato que diferencia e impulsiona a adoção desse instrumento no processo de aprendizagem organizacional.

Conforme ressaltado por Santos (1988), jogos de empresas praticados em conjunto com atividades de aprendizado aumentam a motivação pessoal e o desempenho dos trabalhos acadêmicos.

Diante disso, a aplicação dos jogos de empresas para o aprendizado e desenvolvimento das organizações ganha ênfase com a utilização do ciclo de aprendizagem vivencial (CAV) no qual os participantes passam por cinco fases: vivência, relato, processamento, generalização e aplicação. Esse processo propicia aos envolvidos a compreensão de atividades organizacionais por intermédio da aprendizagem por vivência do jogo.

Aprender fazendo proporciona a resolução de problemas cotidianos por meio de seus próprios recursos, podendo-se, após esse processo, averiguar os resultados das decisões tomadas. Com isso, o aprendizado é interiorizado de maneira duradoura nos indivíduos (FERREIRA, 2000). Contribuindo com essa análise Hansman (2001) afirma:

a problematização da vida cotidiana pode ser inserida no processo de aprendizagem através de capacitações experimentais, na qual os participantes podem aprender e resolver problemas apresentados diariamente.

Nesse sentido, o presente trabalho tem como objetivo averiguar a relevância e a efetividade da aplicação dos jogos de empresas e do ciclo de aprendizagem vivencial, em uma cooperativa agropecuária, para o desenvolvimento das ações dos participantes ante os fatores internos e externos que tangem uma organização cooperativa.

\section{Jogos de empresas}

Gramigna (1994) introduz os jogos de empresas de maneira simples e concisa nos quais a autora os descreve como atividade espontânea, realizada em grupo e organizada a partir de regras que determinam quem e sobre quais condições obterá êxito. As regras trazem instruções para os jogadores de forma que a atividade pode ser executada pelo grupo sem intervenções do facilitador ou instrutor. Já Lacruz (2004), dá ênfase na aplicação empresarial dos jogos ao afirmar que são modelos dinâmicos de simulação os quais apresentam o cotidiano da área empresarial com conteúdo voltado para a tomada de decisão.

Além disso, ainda segundo Lacruz (2004), os jogadores vivenciam uma experiência marcante e lúdica, conectando-se assim ao aprendizado acadêmico, vivências passadas e ambiente empresarial com base em uma representação da realidade não somente de uma empresa, mas também do mercado. 
Os jogos de empresas conseguem agregar em seu repertório funções que estão presentes nas organizações, como o marketing, a produção, o financeiro, etc. A partir dos jogos é possível vivenciar situações que estão presentes no cotidiano das empresas tais como competição e aspectos econômicos e políticos, os quais proporcionam aos ministradores dos jogos a possibilidade de orientar um comportamento empresarial simulado de forma muito parecida à realidade (SAUAIA, 1995).

Sauaia (1995) argumenta ainda que, os participantes têm a oportunidade de assumirem papéis gerenciais, definir livremente metas e aplicar as estratégias. Dessa forma é possível ter um controle dos resultados que foram produzidos pelas metas e estratégias previamente estabelecidas, fazendo com que suas decisões tenham papel decisivo nos resultados.

A partir da utilização dos jogos de empresas, a vivência pode ser alcançada sem a necessidade de experiência, a priori, dos participantes em empresas reais. A vivência é obtida por meio dos erros e acertos, consequências das tentativas sucessivas que moldam as habilidades e comportamentos dos participantes (SAUAIA, 1995).

A possibilidade de um feedback aos participantes e a dimensão temporal fazem com que permita a observação das consequências das decisões tomadas e que se possa aprender com os erros cometidos. Porém, apesar de saber que é possível aprender por meio dos erros em uma simulação, normalmente os professores do ensino tradicional estabelecem seus critérios de aprovação nessas atividades tendo como base o desempenho no jogo, ou seja, a nota dos alunos e o aprendizado deles podem não ser compatíveis (MOTTA; MELO; PAIXÃO, 2012).

Junior et al. (2015) argumentam que os jogos de empresas apresentam uma maior experiência quando comparada a outros métodos de ensino e essa superioridade é maior quando aplicada no desenvolvimento de habilidades gerenciais. Ainda segundo Junior et al. (2015), as maiores dificuldades das instituições de ensino superior em relação à utilização dos jogos de empresas são a falta de profissionais treinados tanto com conhecimento teórico quanto prático, alto valor dos jogos, infraestrutura inadequada e ausência de consciência institucional.

\section{Ciclo de Aprendizagem Vivencial}

De acordo com Vila e Falcão (2002), os jogos de empresas, com objetivo de treinar pessoas, apenas tem sentido quando ao final os participantes adquirem conhecimento suficiente para conseguirem mudar seu comportamento.

Nesse sentido o Ciclo de Aprendizagem Vivencial (CAV) se apresenta como método que incentiva os participantes a se envolverem no processo de solução de um problema, desafio ou atividade. Esse envolvimento faz parte de um processo de ensino-aprendizagem no qual o saber é construído a partir das vivências dos participantes da atividade.

Segundo Lisboa (2004), o CAV é um método desenvolvido com o intuito de tornar sistemática e possível o raciocínio sobre uma atividade na qual o aprendizado seja efetivo e que, por consequência, mude comportamentos.

$\mathrm{Na}$ aprendizagem cooperativa, que pode ocorrer dentro dos jogos de empresa, os participantes adquirem competências e conhecimentos estratégicos. Essa aprendizagem é construída durante o CAV, momento em que os participantes podem perceber que o sucesso ou insucesso da atividade está diretamente relacionado ao sucesso do outro e que as dificuldades podem ser superadas com o trabalho em equipe. Ao realizar o CAV os participantes identificam pontos fortes e fracos sobre as soluções propostas por eles para a situação vivenciada (RIZZO; CAPELLARO; CRISTINI, 2015).

Para que o CAV seja aplicado é necessário que os participantes se envolvam nas suas fases. Nesse sentido, é apresentado abaixo o quadro 1 no qual são descritas as cinco fases do CAV.

O instrutor ou facilitador do processo deve necessariamente conduzir os participantes da atividade de aprendizagem para que estes passem por todas as cinco etapas do CAV, sob pena de distúrbios na aprendizagem do grupo, caso ocorram saltos ou mesmo interrupções antes do completo encerramento de uma fase. 
Quadro 1 - Fases do Ciclo de Aprendizagem Vivencial

\begin{tabular}{ll}
\hline \multicolumn{1}{c}{ Fase } & \multicolumn{1}{c}{ Descrição } \\
\hline 1 - Vivência & $\begin{array}{l}\text { É o jogo propriamente dito, proporciona aprendizado por meio da descoberta. } \\
\text { Momento no qual as pessoas já vivenciaram o jogo e estão prontas para compartilhar o que } \\
\text { perceberam e como sentiram durante o processo. }\end{array}$ \\
3 - Processamento & $\begin{array}{l}\text { Momento no qual os participantes vão associar o que viveram com os padrões de comportamento } \\
\text { no grupo e sistematizar a experiência vivida. }\end{array}$ \\
4 - Generalização & $\begin{array}{l}\text { Nessa fase o ministrante colabora para que os participantes deem um passo na aprendizagem } \\
\text { passando da realidade do jogo para a realidade do seu cotidiano. Nesse sentido, passam a ser } \\
\text { discutidas questões reais e o jogo é deixado de lado. }\end{array}$ \\
5 - Aplicação & Momento de planejar novos rumos e conseguir dos participantes comprometimentos para mudança. \\
\hline
\end{tabular}

Fonte: Elaboração própria, 2016.

\section{Metodologia}

Para realização do presente trabalho, foram elaborados dois questionários $\left(t_{0}\right.$ e $\left.t_{1}\right)$ para averiguar os conhecimentos dos participantes antes e após o treinamento realizado pelos ministrantes do curso, como forma de avaliar os aprendizados alcançados.

O grupo era formado por 12 participantes que responderam os questionários nos dois momentos. Vale ressaltar a heterogeneidade desse grupo, composto por cooperados e funcionários de uma cooperativa. O fato do grupo ser heterogêneo ampliou a efetividade do trabalho, uma vez que, permitiu que o método fosse aplicado com um grupo de origem e formação diversa, porém tendo entre eles uma organização comum.

Os questionários aplicados buscaram informações a respeito do conhecimento dos participantes sobre a existência de conhecimentos prévios a respeito dos jogos de empresas e do CAV. Os participantes foram perguntados também se estariam dispostos a utilizar as metodologias acima para um treinamento ou formação. O quadro abaixo apresenta as questões que foram respondidas pelos participantes.

Para analisar os discursos dos participantes foi utilizado o método qualitativo do discurso do sujeito coletivo por amostra de acessibilidade e conveniência (DSC) com base no questionário do Quadro 2.

Fernando Lefevre e Ana Maria Cavalcanti Lefevre foram os criadores dessa técnica de tabulação e organização de dados no âmbito qualitativo. Foi desenvolvida no final da década de 90 tendo como base a teoria da representação pessoal, que é composta por um compêndio de discursos que tendem a lógicas parecidas, "por meio de procedimentos sistemáticos e padronizados" (FIGUEIREDO; CHIARI; GOULART, 2013).

O DSC apresenta conteúdos com pensamentos de teor de forma igualitária na qual são reunidos em um único discurso em busca de representar todos os indivíduos. São transcritos argumentos realizados pelos participantes tanto de forma oral quanto escrita (FIGUEIREDO; CHIARI; GOULART, 2013).

Os autores apontam ainda que, os discursos do sujeito coletivo como método, compõe um mural de representações sociais, baseado em artifícios metodológicos, que tentam resgatar o pensamento coletivo de forma neutra (FIGUEIREDO; CHIARI; GOULART, 2013).

A tabulação dos dados foi realizada levando em consideração o método DSC através do software MS Excel.

Nos discursos foram verificadas as expressões chaves, a partir delas foram identificadas as ideias centrais (IC) sendo reunidas em um grupo de IC's (IC-1, IC-2, IC3...). De posse das ideias centrais, foi possível construir um discurso único que representa o pensamento coletivo.

Foram retirados dos discursos ruídos ocasionados por ilegibilidade de palavras e expressões fora de contexto. Depois de transcritos os discursos do sujeito coletivo, foram buscadas referenciais na

\section{Quadro 2 - Questionário $\left(\mathrm{t}_{0}\right.$ e $\left.\mathrm{t}_{1}\right)$}

Teste $0\left(t_{0}\right)$ antes do treinamento e teste $1\left(t_{1}\right)$ após o treinamento

1 - Você conhece o que são os jogos de empresas? (Sim ou não)

2 - Você conhece o ciclo de aprendizagem vivencial? (Sim ou não)

3 - Você usaria os jogos de empresas ou o CAV em um treinamento ou formação? Por que? (Sim, justificativa)

Fonte: Elaboração própria, 2016. 
literatura, informações que relacionam o pensamento do coletivo com teóricos especializados na área.

Outro questionário formado por perguntas fechadas foi utilizado para averiguar a qualidade do curso como um todo e da satisfação dos participantes. Esse questionário foi aplicado apenas após o término do curso. Para a análise desses dados utilizou-se o MS Excel.

O treinamento e os questionários foram aplicados para um grupo de 8 cooperados e 4 funcionários na sede da cooperativa no dia 9 de novembro de 2015. Na próxima seção, serão apresentados os resultados com base na metodologia aplicada e no referencial teórico presente ao longo do trabalho.

\section{Resultados e Discussão}

Após a análise dos dados verificou-se que antes do treinamento $\left(\mathrm{t}_{0}\right)$ apenas 3 participantes conheciam o que eram jogos de empresas e nenhum deles conhecia o que era o ciclo de aprendizagem vivencial. Vale ressaltar que os participantes que afirmaram conhecer os jogos de empresa tiveram contato com o mesmo em atividades de formação realizadas previamente, dentro do programa PROEXT de educação cooperativa e capacitação técnica e gerencial das cooperativas agropecuárias das matas de minas..

Após o término do treinamento foi aplicado o segundo questionário contendo as mesmas perguntas $\left(t_{1}\right)$. Os resultados obtidos em $t_{1}$ indicam que todos os participantes passaram a conhecer tanto o que são os jogos de empresas quanto o CAV. Além disso, os participantes manifestaram que utilizariam essas técnicas para um treinamento ou formação em seu grupo de convívio ou mesmo no seu ambiente de trabalho.

A qualidade do trabalho e domínio do assunto por parte dos instrutores/facilitadores, no que se refere tanto à apresentação dos conteúdos, quanto à condução dos jogos e do CAV, são de suma importância para a maximização da interiorização dos aprendizados.

Em função desses fatores, foi realizada uma pesquisa de satisfação que verificou a qualidade do curso. Os resultados são apresentados nas figuras 1 a 4.

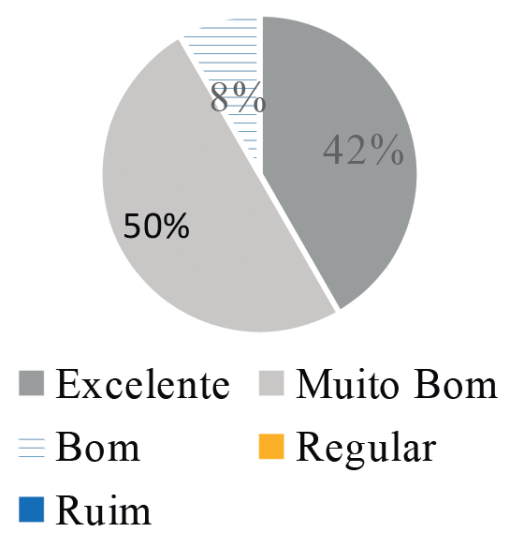

Figura 1 - Domínio dos ministrantes do curso a respeito do assunto tratado Fonte: Elaboração própria, 2016.
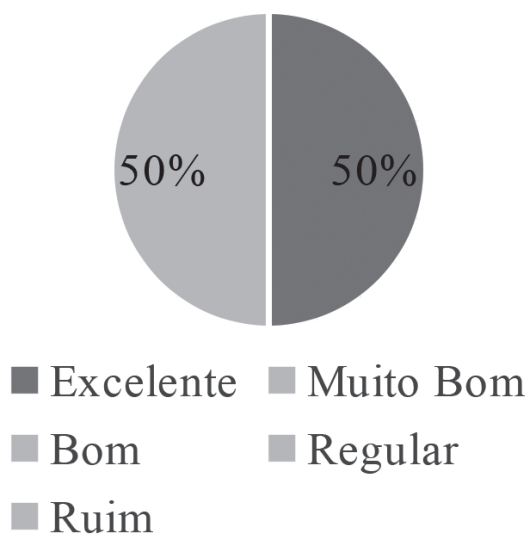

Figura 2 - Qualidade do conteúdo e material didático Fonte: Elaboração própria, 2016. 
Após análise dos questionários, percebe-se que o nível de satisfação a respeito das atividades realizadas, no geral, foi positivo. A grande maioria dos participantes escolheu como resposta "Excelente" e "Muito Bom" o que demonstra, além da relevância, a qualidade do curso na opinião dos participantes.

Foram identificadas 8 ideias centrais a partir das respostas dos participantes. É importante ressaltar que, embora tenham sido realizados 12 questionários, os discursos podiam apresentar mais de uma ideia central, o que fez com que a frequência acumulada supere o número de discursos. As principais informações sobre as IC's estão apresentadas na tabela abaixo.

Em razão da grande homogeneidade das respostas foram selecionadas as 6 ideias centrais com maior presença nos discursos, já que essas apresentaram porcentagens elevadas no Perc. 2. As próximas tabelas apresentam os discursos do sujeito coletivo, derivadas das 6 IC's comentadas anteriormente.
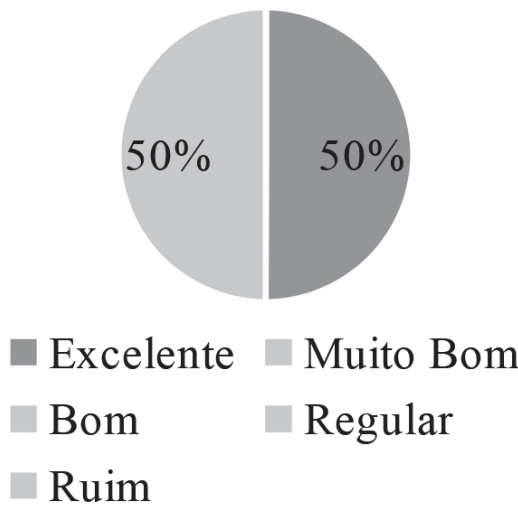

Figura 3 - Nível de satisfação com o treinamento

Fonte: Elaboração própria, 2016.

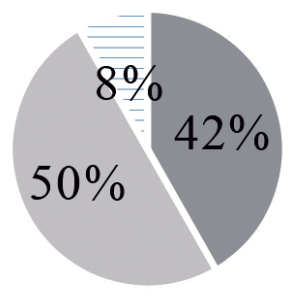

\section{Excelente Muito Bom \\ EBom Regular \\ Ruim}

Figura 4 - Incentivo a participação e esclarecimento de dúvidas

Fonte: Elaboração própria, 2016.

Tabela 1 - Frequência acumulada por ideia central

\begin{tabular}{|c|c|c|c|c|c|}
\hline & Ideia Central & Quant. & Freq. Ac. & Perc. $1^{*}$ & Perc. $2^{* *}$ \\
\hline IC-1 & $\begin{array}{l}\text { Aprendizado da metodologia CAV e de jogos de empresas como } \\
\text { conteúdo prático. }\end{array}$ & 7 & 7 & $18 \%$ & $58 \%$ \\
\hline IC-2 & Vivência, aplicação das metodologias no dia a dia. & 6 & 13 & $16 \%$ & $50 \%$ \\
\hline IC-3 & Cotidiano, percepção das práticas vivenciadas nas atividades diárias. & 6 & 19 & $16 \%$ & $50 \%$ \\
\hline IC -4 & $\begin{array}{l}\text { Importância do planejamento para a execução e alcance dos } \\
\text { resultados estabelecidos a priori. }\end{array}$ & 6 & 25 & $16 \%$ & $50 \%$ \\
\hline IC-5 & $\begin{array}{l}\text { Relevância da participação e de atividades coletivas para a } \\
\text { maximização de resultados. }\end{array}$ & 5 & 30 & $13 \%$ & $42 \%$ \\
\hline IC- 6 & $\begin{array}{l}\text { Visualização da dimensão de uma boa negociação por meio } \\
\text { dos jogos de empresas. }\end{array}$ & 4 & 34 & $11 \%$ & $33 \%$ \\
\hline IC-7 & Importância do processo do conhecimento & 2 & 36 & $5 \%$ & $17 \%$ \\
\hline IC-8 & Ouvir com maior frequência as demais pessoas. & 2 & 38 & $5 \%$ & $17 \%$ \\
\hline Total & & 38 & 38 & $100 \%$ & \\
\hline
\end{tabular}

*Quantidade de IC's nos discursos dividida pela freq. acumulada total.

**Quantidade de IC's nos discursos dividida pelo número de total de discursos.

Fonte: Elaboração própria, 2016. 
Junior et al. (2015, p.2) argumenta que, por meio do ensino tradicional, os alunos não podem ter sentimento de frustração e prazer reais das profissões dado que estes só são alcançados por meio da prática. Somado a isso, ele argumenta que o meio acadêmico aborda "em seu conteúdo do currículo básico, somente as partes automatizadas de um todo, tratadas em separado, como detalhes de um sistema complexo e dinâmico, esquartejado em nome da simplificação educacional, (...)" .

Hilgard (1973) complementa dizendo que, o que é feito dentro da sala de aula nem sempre é possível realizar nas empresas. Em condições de laboratório é possível cometer enganos permitindo que os alunos também os cometa sem maiores malefícios. Segundo Lopes (2001), os jogos de empresas proporcionam melhor experiência quando comparada a outros métodos de ensino, pois leva a vivência empresarial para a sala de aula.

A parceria com a cooperativa em estudo, que resultou em uma participação ativa de seus funcionários e associados no desenvolvimento das atividades prevista no projeto PROEXT, fortaleceu os argumentos apresentados por Junior et al. (2015), de que os jogos de empresas são eficazes, pois conseguem aproximar a teoria e a prática, além de desenvolver habilidades gerenciais e oferecer aos participantes uma visão geral da organização, o que facilita a tomada de decisões estratégicas.

A partir do discurso coletivo, pode-se observar a importância da aplicação dos jogos, ao provocar percepções sobre a relevância do conteúdo aprendido no cotidiano dos participantes. Para Kolb (2014), a aprendizagem vivencial considera o aprendizado como um processo, no qual o conhecimento é produzido por intermédio da transformação da experiência.

Além disso, de acordo com Sauaia (1989), essa vivência facilita o aumento do conhecimento com as incorporações de informações trazidas do contexto do jogo, resgatando conhecimentos anteriormente adquiridos e integrando com novos. Todo esse processo colabora na compreensão dos participantes. Essa situação ficou evidenciada após aplicação dos jogos e a utilização do CAV, permitindo que os participantes compartilhassem seus aprendizados e como as atividades vivenciadas podem ser relevantes em seus devidos contextos.

A partir dos discursos do sujeito coletivo, fica clara a relação das atividades que foram realizadas no treinamento e o dia a dia dos participantes.

A vivência faz com que os participantes se relacionem com as forças de um ambiente real como: estruturas legais, sociais e políticas. Atuando como um orientador para possíveis comportamentos em empresas reais. Com os jogos, é possível que os participantes consigam trabalhar melhor sob pressão, administrar melhor o tempo, otimizar a execução de tarefas. Nesses experimentos, o participante pode ousar mais, podendo até durante o jogo incorrer em perdas de milhões de reais ou tomar atitudes que não agradariam acionistas, fazendo-se, assim, que ocorra um aprendizado para que não ocorra em situações reais (JUNIOR et al., 2015).

\section{Quadro 3 - Ideia central 1 - Aprendizado}

Ideias central: IC-1

Aprendizado da metodologia CAV e de jogos de empresas como conteúdo prático.

Discurso do Sujeito Coletivo:

O ciclo de aprendizagem vivencial e os jogos de empresas possibilitaram uma grande quantidade de informações interiorizadas, aprendidas, sendo estas voltadas para situações práticas. Pode-se perceber o maior nível de conhecimento gerado, sendo preferida quando comparada a outros métodos do ensino tradicional. O ciclo de aprendizagem vivencial e os jogos de empresas propiciaram uma forma de ensino dinâmica na qual a participação e interação são constantes. Estes dois métodos de ensino estimulam a formação de estratégias para melhorar os resultados a serem obtidos. Os conteúdos aprendidos durante o repasses de metodologia ao longo do curso são altamente aplicáveis em situação do dia a dia, facilitando a transição do conhecimento apenas teórico para um mais amplo de teoria somado a prática.

Fonte: Elaboração própria, 2016.

Quadro 4 - Ideia Central 2 - Vivência

Ideias central: IC-2

Vivência, aplicação das metodologias no dia a dia.

Discurso do Sujeito Coletivo:

Com base na metodologia do ciclo de aprendizagem vivencial e dos jogos de empresas, torna-se possível a aplicação da experiência adquirida no cotidiano. As metodologias aplicadas possibilitaram viver várias experiências que são apresentadas a nós em nosso dia a dia e nos ajudou a ter mais experiências para enfrentá-las com mais habilidade e segurança. Pois a partir do momento em que se coloca o conteúdo aprendido no curso em prática, as quantidades de informações facilitam nos processos de trabalho. Por meio da dinâmica apresentada conseguimos elaborar e entender as soluções propostas para o dia a dia.

Fonte: Elaboração própria, 2016. 
Quadro 5 - Ideia Central 3 - Aplicação no cotidiano

Ideias central: IC-3

Cotidiano, percepção das práticas vivenciadas nas atividades diárias.

Discurso do Sujeito Coletivo:

A partir das situações criadas pela metodologia CAV por meio dos jogos de empresas foi possível enxergar situações diretamente relacionadas ao nosso cotidiano. Antes, por muitas vezes, elas passavam despercebidas e não se buscava métodos de ajuda. Percebe-se que é essencial em nosso meio, ou seja, na vida real e é altamente aplicável nos processos do trabalho. Com o curso, podese atentar melhor para os pontos fracos e, assim, aplicar um empenho maior nessas tarefas, as quais estão claramente interligadas ao dia a dia.

Fonte: Elaboração própria, 2016.

\section{Quadro 6 - Ideia Central 4 - Importância do Planejamento}

Ideias central: IC-4

Importância do planejamento para a execução e alcance dos resultados estabelecidos a priori.

Discurso do Sujeito Coletivo:

A realização do ciclo de aprendizagem vivencial e dos jogos de empresa, permitiu que os participantes se atenham às necessidades da realização do planejamento para o alcance dos objetivos almejados. As metodologias possibilitaram a compreensão da importância de administração do tempo para consecução das tarefas. Pois o planejamento permite o entendimento das ações a serem traçadas, facilitando na forma de analisar e agir.

Fonte: Elaboração própria, 2016.

Ao aplicar o ciclo de aprendizagem espera-se que os integrantes reflitam sobre questões presentes no contexto dos mesmos, atingindo questões pessoais ou diretamente ligadas ao ambiente de trabalho, como relatado no discurso acima.

Os jogos possibilitam o enfrentamento de questões pelos quais os participantes devem tomar decisões estratégicas que terão consequências negativas ou positivas para o alcance do objetivo estabelecido, sendo esse um momento propício ao erro, por possibilitar, após a realização dos jogos, reflexões sobre as ações tomadas e o que elas podem representar na realidade de cada participante.

De acordo com Junior et al. (2015, p.9) “o jogo de empresas é uma ferramenta que estimula a estratégica organizacional". Provocando nas pessoas a compreensão e reflexão da importância das atividades que fazem parte da instituição e de como elas podem estar agindo ou o que podem propor para o desenvolvimento delas.

Por uma consistente simulação do ambiente empresarial, os jogos de empresas conseguem maior difusão, instigando a aprendizagem vivencial, na qual o participante é o ator principal e por meio de suas próprias experiências adquire aprendizado (NEVES; LOPES, 2008).

De acordo com Sauaia (1995) os jogos de empresas são técnicas apropriadas para o desenvolvimento de habilidades por meio da prática continuada da resolução de problematizações e também para a interiorização de atitudes em função dos estímulos à tomada de decisões em grupos, em um ambiente que se parece com o dia a dia.

No que concerne ao trabalho em grupo, o trabalho cooperativo faz com que maiores possibilidades e ideias sejam encontradas, as quais não seriam possíveis de serem alcançadas com o aprendizado individual (BROWN; COLLINS; DUGUID, 1989).

Para Lacruz (2004) as definições apresentadas coincidem no argumento de que jogos de empresa são modelos dinâmicos de simulação que salientam as situações da área empresarial, bem como o aspecto sequencial.

De acordo com Lopes (2001) os jogos de empresas oferecem uma experiência superior quando comparados a outros métodos de ensino, primordialmente nos quesitos de habilidades gerenciais. Sendo que esse pensamento é evidenciado com a aplicação da metodologia e nele é possível ampliar as visões perante determinados assuntos que tangem uma organização.

Um dos pontos favoráveis na aplicação de jogos de empresa está relacionado ao estímulo do pensamento sistêmico, proporcionando aos integrantes do curso uma maior compreensão em especificidades do sistema, como a negociação apresentada no discurso coletivo, fazendo com que esses integrantes possam analisar, agir ou até mesmo interferir em ações futuras de acordo com o que foi aprendido (NEVES; LOPES, 2008). 
Quadro 7 - Ideia Central 5 - Relevância da Participação

Ideias central: IC-5

Relevância da participação e de atividades coletivas para a maximização de resultados.

Discurso do Sujeito Coletivo:

O ciclo de aprendizagem vivencial e os jogos de empresas são ferramentas que propiciam os participantes atuarem em grupos, dinâmica essa que facilita a aprendizagem de situações reais. Percebe-se a importância do envolvimento de todos no processo. Dessa forma, refutase o pensamento, exclusivamente, individualista em detrimento do pensamento coletivo. Com isto, constata-se que o trabalho coletivo é aquele que gera melhores resultados.

Fonte: Elaboração própria, 2016.

\section{Quadro 8: Ideia Central 6 - Importância da Negociação}

Ideias central: IC-6

Visualização da dimensão de uma boa negociação por intermédio dos jogos de empresas.

Discurso do Sujeito Coletivo:

Em decorrência do ciclo de aprendizagem vivencial e dos jogos de empresas, foi construído um entendimento no que tange uma negociação. $\mathrm{O}$ curso possibilitou a compreensão sobre formas de negociação que incidem tanto nas atividades empresariais, quanto num relacionamento interpessoal.

Fonte: Elaboração própria, 2016.

\section{Considerações Finais}

Este trabalho teve o intuito de averiguar a relevância e a efetividade da aplicação do jogo de empresas e a utilização da metodologia do ciclo de aprendizagem vivencial, em uma cooperativa agropecuária do estado de Minas Gerais.

Para isso, foi utilizado o método DSC como forma de interpretação dos resultados. Esse método permitiu sintetizar as informações dos participantes referentes à prática dos jogos de empresas e do CAV ao transmitir as concepções das atividades realizadas por meio de discursos.

Por intermédio do curso, os participantes tiveram a oportunidade de trabalharem com questões pertencentes ao ambiente organizacional que, por muitas vezes, passavam despercebidos ou não recebiam a devida atenção.

Dessa forma, o jogo de empresas contribuiu para a aprendizagem de algumas das atividades, como as de planejamento estratégico, negociação, gestão do tempo, a importância da participação e o do trabalho em equipe, referentes ao sistema do qual fazem parte, possibilitando aos cooperados e funcionários novas maneiras de interpretar e agir de acordo com ações decorrentes do ambiente externo e interno das organizações. O ciclo de aprendizagem vivencial possibilitou aos integrantes compartilharem o conhecimento adquirido com a aplicação do jogo de empresas e demonstrarem seus diferentes pontos de vistas e sentimentos sobre as atividades realizadas, ao se criar um ambiente de interação e espontaneidade fazendo com que os participantes ficassem à vontade para expor suas opiniões, desenvolvendo um conhecimento compartilhado entre os mesmos, enriquecendo o curso ao construir dessa maneira um aprendizado coletivo.

Durante todo o treinamento foi enfatizada a importância dos conteúdos, teórico e prático, apresentados para que os participantes os aplicassem em sem seus contextos.

\section{Agradecimentos:}

Os autores agradecem a todos os participantes do projeto de extensão "Programa de Educação Cooperativa e Capacitação Técnica e Gerencial das Cooperativas Agropecuárias das Matas de Minas", cuja colaboração e apoio permitiu a elaboração deste artigo. Este projeto de extensão foi financiado com recursos do PROEXT/MEC.

\section{Referências}

BROWN, J. S.; COLLINS, A.; DUGUID, P. Situated Learning and the Culture of Learning. Education Researcher, v. 18, n. 1, p. 32-42, 1989.

FERREIRA, J. Â. Jogos de empresas: Modelo para aplicação prática no ensino de custos e administração do capital de giro em pequenas e médias empresas industriais. (Doutorado em Engenharia da Produção), UFSC, 2000. Disponivel em < https://repositorio.ufsc.br/bitstream/handle/123456789/78704/ 184957.pdf? sequence=1>. Acesso em: 6 abr. 2016. 
FIGUEIREDO, M. Z. A.; CHIARI, B. M.; GOULART, B. N. G. DE. Discurso do Sujeito Coletivo/ : uma breve introdução à ferramenta de pesquisa qualiquantitativa. Comunicação, v. 25, n. 1, p. 129-136, 2013.

GRAMIGNA, M. R. M. Jogos de Empresa. Markron Books, 1994.

HANSMAN, C. A. Context-based adult learning. New directions for adult and continuing education, v. 43-52, 2001.

HILGARD, E. R. Teorias da aprendizagem. Ed. Pedagógica e Universitária, 1973.

JUNIOR, A. G. et al. A Contribuição dos Jogos de Empresas no Aprendizado sobre Estratégia. SEMEAD, 2015.

KOLB, D. A. Experiential learning: Experience as the source of learning and development. FT Press, 2014.

LACRUZ, A. J. Jogos de empresas: considerações teóricas. Caderno de Pesquisas em Administração, p. 93-109, 2004.

LISBOA, R. Jogos empresariais. Ebooks, 2004. Disponivel em <http://www.mettodo.com.br/ebooks/ Jogos\%20Empresariais.pdf $>$. Acesso em: 30 mar. 2016.

LOPES, P. DA C. A perspectiva do animador com a utilização na pós-graduação lato sensu. ENANPAD - Encontro da Associação Nacional de Pós-graduação e Pesquisa em Administração, 2001.

MOTTA, G. D. S.; MELO, D. R. A. DE; PAIXÃO, R. B. O jogo de empresas no processo de aprendizagem em administração: o discurso coletivo de alunos. Revista de Administração Contemporânea, v. 16, n. 3, p. 342-359, 2012.

NEVES, J. P.; LOPES, C. Jogos de Empresas: Um Estudo da Utilização em Cursos de Graduação em Administração no Estado de São Paulo. ENANPAD - Encontro da Associação Nacional de Pós-graduação e Pesquisa em Administração, 2008.

RIZZO, S.; CAPELLARO, F.; CRISTINI, V. Flipped classroom, LCA y materiales de contrucción: una experiencia didática para una actividad de aprendizaje cooperativa cooperativa y activa. III Workshop on Educational Innovation in Architecture (JIDA'15), 2015.

SANTOS, Theobaldo Miranda. Noções de Prática de Ensino. São Paulo: Companhia Editora Nacional, 1988.

SAUAIA, A. C. A. Jogos de empresas: tecnologia e aplicação. Revista de Administração da USP, São Paulo, v.32, no3, p.13-27, julho/setembro 1997.

SAUAIA, A. C. A. Satisfação e Aprendizagem em Jogos de Empresas: Contribuições para a Educação Gerencial. (Doutorado em Finanças e Marketing) - Faculdade de Economia, Administração e Contabilidade, Universidade de São Paulo. 1995. Disponível em <http://www.teses.usp.br/teses/ disponiveis/12/12134/tde-23112005-193556/pt-br.php>. Acesso em: 4 abr. 2016.

VILA, M.; FALCÃO, P. Focalização de Jogos em TED. Rio De Janeiro: Qualitymark Editora, 2002.

Recebido para publicação em 30/4/2016 e aprovado em 15/6/2016. 